\title{
White CdS Nanoluminophore based Tunable Hybrid Light Emitting Diodes
}

\author{
Sedat Nizamoglu, Evren Mutlugun, Ozgun Akyuz, Nihan Kosku Perkgoz, Hilmi Volkan Demir \\ Department of Physics, Department of Electrical and Electronics Engineering, Nanotechnology Research Center, \\ Bilkent University, Ankara, 06800 Turkey \\ Tel:[+90]( 312) 290-1021 E-mail: volkan@bilkent.edu.tr
}

\section{Lydia Liebscher, Sameer Sapra, Nikolai Gaponik, Alexander Eychmüller \\ Physikalische Chemie, TU Dresden, Bergstr. 66b, 01062 Dresden, Germany}

Significant progress in nanocrystal based device applications, especially in white light emitting diodes (WLED), has been achieved in recent years ${ }^{1-10}$. White light generation using $\mathrm{CdSe} / \mathrm{ZnS}$ core-shell nanocrystals (NCs) of single, dual, trio, and quadruple combinations hybridized with blue InGaN/GaN LEDs has been shown ${ }^{1-2}$. White light generation with high color rendering index with dual hybridization of polymers and nanocrystals has also been realized ${ }^{4}$. Additionally, color rendering index higher than 90 has been attained using only a single type of conjugated polymers hybridized on nitride LEDs ${ }^{5}$. Utilization of a blue/green two-wavelength InGaN/GaN LED coated with a single type of red NCs as well as a blue InGaN/GaN LED with a single type of yellow NCs and a dual type of red and green NCs have been reported ${ }^{6-7}$. Furthermore, a white LED has also been fabricated by coating a blended mixture of CdSeS NCs with polymethylmethacrylate (PMMA) on a near-UV (n-UV) LED ${ }^{8}$ and using layer-by-layer assembly of CdSe/ZnS NCs on a n-UV LED,10. For the current state-of-the-art, quantum dot based hybrid white light generation is achieved using the collective photoluminescence from the combination of different types of nanocrystals or using the luminescence arising from the combination of nanocrystals and LED platform. However, in these approaches one is forced to engineer the hybrid device parameters such as the type and concentration of NCs as well as the thickness and order of the NC films at the device level. To overcome this problem, white nanoluminophore particles are used to generate white light directly at the quantum dot level. For example, wellknown magic-sized CdSe nanocrystals with a diameter of 1-2 $\mathrm{nm}$ generate white light, but their quantum efficiency (QE) is restricted to $2-3 \%{ }^{11}$. Different from CdSe nanocrystals, CdS surface state emitting (SSE) nanocrystals with a dot diameter of 2-3 $\mathrm{nm}$ generate white light exhibiting a relatively higher quantum efficiency of $17 \%{ }^{11}$. Moreover, by using only $\mathrm{CdS}$ nanoluminophores it is possible to achieve white light generation with a color rendering index of more than 70. On the other hand, when only one type of yellow NC is hybridized on blue LED, white light generation has been possible with a color rendering index of $14.6^{2}$. Therefore, the use of white CdS nanocrystal luminophores for hybrid device application provides important benefits in terms of simpler device engineering, relatively higher quantum efficiency, and color rendering index.

In this work, we present high-quality white light generation using the hybridization of white nanoluminophore CdS crystals on InGaN/GaN n-UV light emitting diodes. We demonstrate that the color properties of these hybrid white LEDs are tuned with controlling the number of their hybridized NCs. Figure 1(a) shows the operating $(x, y)$ coordinates of these tuned hybrid light sources on CIE 1931 chromaticity diagram ${ }^{12}$.

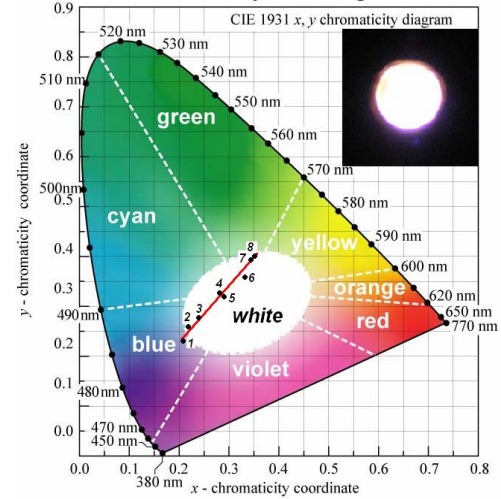

(a)

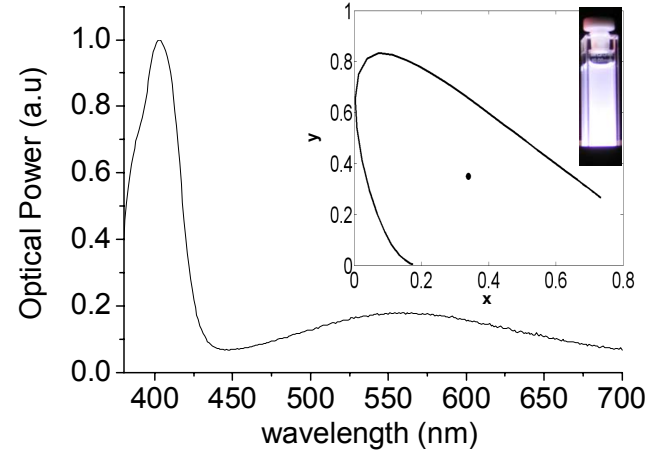

(b)

Figure 1: (a) (x,y) chromaticity coordinates of hybrid white LEDs based on CdS surface state emitting nanocrystal luminophores (inset: photograph of the resulting WLED while emitting white light when electrically driven) and (b) photoluminescence spectrum of these CdS nanoluminophores in toluene along with its corresponding (x,y) chromaticity coordinate (inset: photograph of CdS nanoluminophores in solution under UV excitation). 
For white light generation we use trap-rich CdS nanocrystals. The surface states of these NCs give rise to a broad emission band that spans the entire width of the visible spectrum as shown in Fig. 1(b). The photoluminescence (PL) of these SSE CdS NCs in toluene solution depicted in Fig. 1(b) corresponds to the chromaticity coordinate of $(\mathrm{x}=$ $0.34, \mathrm{y}=0.35$ ), the correlated color temperature of $5146 \mathrm{~K}$ and the color rendering index of 82.5. All hybrid devices (Sample 1 to 8) are implemented using CdS nanocrystals (in multiples of $45.2 \mathrm{nmol}$ ) on $\mathrm{InGaN} / \mathrm{GaN}$ based LED pump sources with a peak wavelength of $383 \mathrm{~nm}$ in the n-UV. The MOCVD epitaxial growth and fabrication procedures described elsewhere ${ }^{13}$ are used. Here the operating principle of these hybrid NC-WLEDs relies simply on the hybrid use of the n-UV LED as the high energetic photon source to excite nanoluminophore CdS particles and these nanoluminophores to generate white light.

By controlling the number of surface state emitting NCs hybridized on n-UV LED platform, we achieve tunability of optical properties such as the $(\mathrm{x}, \mathrm{y})$ chromaticity coordinates, color temperature $\left(\mathrm{T}_{\mathrm{c}}\right)$ and color rendering index CRI $\left(R_{a}\right)$ as summarized in Table 1. When the number of hybridized SSE nanocrystals increases, the $x$ - and y-axis of the chromaticity coordinate increases and the correlated color temperature decreases as illustrated in Table 1 . There are different mechanisms that lead to this tuning behavior with the varying number of NCs. First, as the NC number increases, the reabsorption of the generated photons by lower energy states on the quantum dot increases. After the high energetic photons are absorbed by the trap states of these NCs, they generate photons with the corresponding colors from violet to red. Since the trap states in the red range can absorb the photons with energy less than red, the probability of the reabsorption is observed to increase proportionally with the number of nanocrystals due to the fact that one photon can encounter with many trap states having lower energies. Second, another important mechanism is the non-radiative energy transfer (ET). In this case, after an electron-hole pair is generated due to absorption, it does not recombine in that trap state but it recombines at a trap state on another $\mathrm{NC}$ with a lower energy gap, which can result with a red shift in the visible spectrum. Additionally, since the nanocrystals have a size distribution, as a result of the non-radiative ET, the recombination transitions would occur on the nanocrystals with larger sizes whose band gap is smaller. We observe the non-radiative recombinations by looking at the interband transition peaks of $\mathrm{CdS}$ NCs. For the in-solution interband transition, PL peak wavelength is detected at $403.0 \mathrm{~nm}$, but when the NCs are embedded into a solid PMMA thin film, for instance as in Sample 8, the PL peak wavelength shifts to $414.7 \mathrm{~nm}$ because of the recombination in larger dots as a result of the non-radiative ET.

In conclusion, we fabricated and demonstrated hybrid white light emitting diodes that integrate white emitting CdS nanoluminophores with high light quality $(\mathrm{CRI}>70)$. We also showed that the resulting white light properties of these hybrid WLED (including $(\mathrm{x}, \mathrm{y})$ chromaticity coordinates, color temperature, and color rendering index) are conveniently tuned as desired across the white region by controlling the number of these surface state emitting nanoluminophores hybridized on the n-UV LED platform. In this tuning process, with the increasing number of the hybridized SSE NCs, we observed that $(x, y)$ and $R_{a}$ increase while $T_{c}$ decreases.

Table 1: The optical properties of hybrid WLEDs

\begin{tabular}{lllll}
\hline Sample & $\mathrm{x}$ & $\mathrm{y}$ & $\mathrm{T}_{\mathrm{c}}(\mathrm{K})$ & $\mathrm{R}_{\mathrm{a}}$ \\
\hline 1 & 0.21 & 0.23 & 34463 & 57.3 \\
2 & 0.22 & 0.26 & 30159 & 62.1 \\
3 & 0.24 & 0.28 & 16174 & 66.3 \\
4 & 0.28 & 0.33 & 8259 & 72.6 \\
5 & 0.29 & 0.32 & 7586 & 73.2 \\
6 & 0.33 & 0.36 & 5564 & 73.4 \\
7 & 0.34 & 0.39 & 5154 & 74.6 \\
8 & 0.35 & 0.40 & 4718 & 74.9
\end{tabular}

Acknowledgements: This work is supported by EU Network of Excellence PHOREMOST, project 511616 and Marie Curie European Reintegration Grant MOON 021391 within the 6th European Community Framework Program and TUBITAK under the Project No. EEEAG 106E020, 104E114, 105E065, and 105E066. HVD and SN also acknowledge additional support from the Turkish National Academy of Sciences Distinguished Young Scientist Award and TUBITAK Fellowship programs.

\section{References}

[1] S. Nizamoglu et al., Nanotechnology, 18065709 (2007).

[2] S. Nizamoglu et al., IEEE COMMAD WO-A5 (2006).

[3] E. Mutlugun et al., Opt. Exp., 151129 (2007).

[4] H. V. Demir et al, New Jour. of Phys., submitted (2007).

[5] I. O. Huyal et al, CLEO CMO5 (2007).

[6] H. Chen et al., IEEE Photon. Technol. Lett., 181430 (2006).

[7] H. Chen et al., IEEE Photon. Technol. Lett., 18193 (2006).
[8] M. Ali et al., Nanotechnology, 18075401 (2007).

[9] S. Nizamoglu et al., J. Opt. A: Pure Appl. Opt., accepted (2007).

[10] S. Nizamoglu et al., Nanomeeting Fri-3 (2007).

[11] S. Sapra et al., Adv. Mater., 19569 (2007).

[12] E. F. Schubert, Cambridge University Press (2006)

[13] E. Sari et al, App. Phys. Lett., 90011101 (2007). 